\title{
APPLY EDGE INTELLIGENCE TO IOT BASED HOME AUTOMATION
}

\author{
Yi Hua Wu and Hongli Luo \\ Purdue University Fort Wayne \\ Fort Wayne, Indiana, USA
}

\begin{abstract}
There are many challenges in building a low-delay IoT system because the reliance on underlying network infrastructure and data analytics in cloud environment. Deep learning combined with edge computing makes edge intelligence possible in IoT system. With deep learning, complicated task including facial recognition and voice control can be processed at edge devices such as mobile phone and microcontroller. In this paper, we present a design and implementation of home automation system which applies deep learning techniques at low-power IoT edge devices. We implemented the facial recognition in TensorFlow Lite on Raspberry Pi. The performance of TensorFlow and TensorFlow Lite on Raspberry Pi is also compared. The experimental data we collected from the system proves that the performance can be efficiently improved using deep learning models in TensorFlow Lite. With all data constrained at local area network, it can reduce processing delay and computation resource consumption, while at the same time provide real-time response and better privacy.
\end{abstract}

\section{KEYWORDS}

Internet of Things, Edge Computing, Deep Learning, Video Surveillance, Privacy

\section{INTRODUCTION}

Internet of Things (IoT) applications have grown rapidly in recent years in areas such as home automation, smart city, health care, asset tracking, and smart manufacturing. There are many challenges to IoT application. The processing of data relies on centralized cloud environment, which needs the support of reliable network infrastructure to connect to Internet. There are concerns over privacy because of the storage and processing of data in cloud. Off-loading the computation intensive tasks to closer to data source and end user can help address those challenges (Wang 2020). Edge computing can reduce the transmission of large volume of IoT data generated by large number of devices from various sensors, cameras, and mobile phones. There are several advantages of edge computing, including reducing latency and resource consumption and providing privacy.

Besides the traditional sensor data, images and audio provide a rich source of IoT data. Deep learning has been advanced significantly in recent years especially in computer vision and natural language processing. It has generated lots of applications, such as video processing, object recognition, self-driving cars, and robotics. Edge intelligence is becoming possible because of the advances of AI and deep learning. Applying AI to edge devices can avoid relying on Internet connectivity, thus reducing bandwidth requirements and resource consumption. In this paper we implemented a low-cost and low-delay home automation system. The goal is to apply state-of-art machine learning technology to provide real-time facial recognition and voice control in IoT system, which are of importance to privacy in a smart home or health care environment. Most of the facial recognition application running on Raspberry Pi using deep learning technology is implemented using TensorFlow. We implemented facial recognition using the most recently introduced TensorFlow Lite. After comparing the performance with TensorFlow, we found that TensorFlow Lite is suitable for low-power devices since it can reduce the resource consumption and achieve a better performance.

The remainder of the paper is organized as follows. Section 2 introduces the background research. Section 3 presents the design and performance of the home automation system. Conclusions and future works are presented in Section 4. 


\section{BACKGROUND RESEARCH}

\subsection{Deep Learning}

Deep learning has been successfully used for computer vision application, natural language processing and speech recognition. There are some cloud machine learning platforms providing APIs for image classification, object detection and natural language processing, for example Google Vision API (2020). To use Google Vision API, you need to upload the images to cloud and then Google AI will analyze the images and return the results. The delay is resulted from transmitting data back and forth between end system and cloud server, in addition to the processing time at Google cloud server. Voice control and gesture recognition provide touchless or hands-free capabilities which is especially important for senior or disabled people. With voice recognition, users can issue simple voice commands to turn on and turn off switches in the home. Edge device can give an immediate response under urgent situation to ensure safety. Amazon's Alexa, Apple's Siri, and Google Assistant are all cloud platforms that provide voice control services.

Training a deep neural network from scratch is time consuming. Transfer learning is a widely used technique to build accurate model efficiently. It reduces the training time for a neural network model while achieving a higher accuracy. The retrained model can be used for inference in resource constrained environments such as mobile phones and embedded devices. To apply transfer learning in computer vision area, a high performance pretrained model for image recognition is selected first. Some of the most popular pretrained models are VGG-16 (Simonyan 2014), Inception (Szegedy, 2015), ResNet (Graves 2013), and MobileNet (Howard 2017). The model will then be retrained with the dataset of our own images for customized image classification or object recognition. Transfer learning can also be used for voice recognition. It can be used to build custom voice recognition model to recognize different words which can be used to control IoT devices.

\subsection{Edge Intelligence for IoT}

With the advance of deep learning, low-cost and high-accuracy image classification and facial recognition system can be running on edge IoT devices. Multiple deep learning frameworks designed for edge computing are now available. TensorFlow (2020) is an end-to-end open source machine learning platform introduced by Google. TensorFlow Lite (2020) is an optimized framework designed to perform machine learning on low power and resource constrained devices such as mobile phone, wearable devices, and microcontrollers, which avoids the transmission of data to a cloud server. Microcontrollers are low power IoT edge devices with limited computation resources that directly interact with sensors and actuators in an IoT system. TensorFlow Lite for Microcontroller was introduced recently on microcontrollers to process tasks like image classification, wake word detection with voice input, and gesture classification. For example, Arduino Nano 33 BLE Sense (2020) is an AI enabled board with various sensors which can run machine learning models using TensorFlow Lite. Besides computer vision related tasks, it also provides voice recognition to recognize simple voice commands.

\section{DESIGN OF THE SYSTEM AND PERFORMANCE EVALUATION}

In this section we present the design and implementation of the IoT home automation systems. To study the performance of applying TensorFlow Lite for facial recognition on edge device, experimental data is collected and presented for analysis.

\subsection{System Design}

Figure 1 displays the components and information flow of the home automation system. The system is composed of sensors and controls, surveillance, data storage and analytics, and application software. At the edge of this IoT system, sensors and actuators are used to interact with physical devices. Various sensors, 
such as temperature-humidity sensor DHT 11, are used to monitor the home environment. In addition to sensors, camera and microphone are also integrated into the IoT system to collect image and voice input from the environment. Data collected by sensors and camera can be wirelessly transmitted to a local gateway server or Internet cloud server using Wi-Fi or Bluetooth technologies for storage and further analytics. User can view the information of home automation system or issue commands to control switches or actuators via web application, Android app.

Figure 2 displays some functionalities implemented in Android app. For example, it can display local weather forecast, turn on and turn off lights of different colors, visualize and analyze history data. User can also watch live surveillance video streaming and view pictures taken by camera. Temperature and humidity data generated by sensor is sent to server via Arduino microcontroller. Mobile app fetches sensor data and images from the database through JSON file. User can issue commands through Android phone to IoT system either through app GUI or voice input. Voice input is processed by calling Android RecognizerIntent API and converted into text commands to control IoT devices.

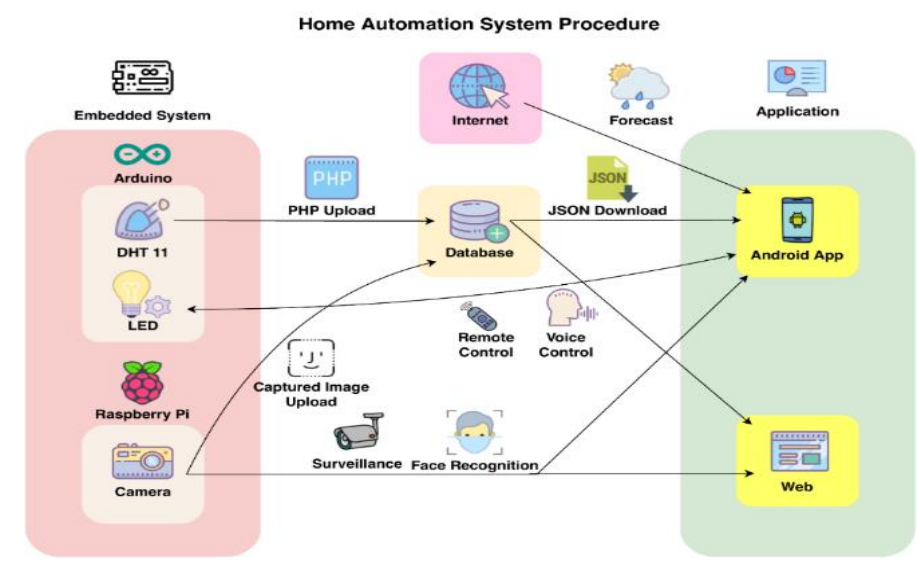

Figure 1. Information Flow of Home Automation System

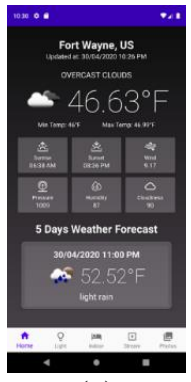

(a)

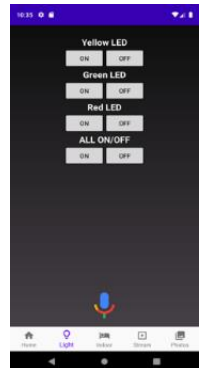

(b)

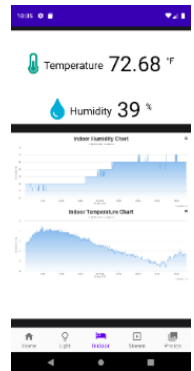

(c)

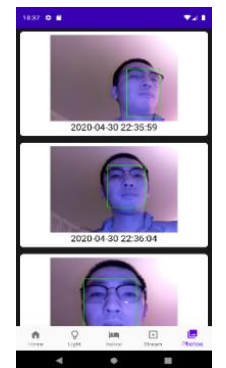

(d)

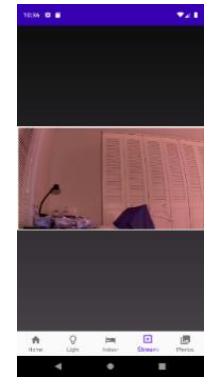

(e)

Figure 2. Android App GUI Design

Home surveillance system is composed of video streaming and facial recognition, both are implemented on a Raspberry Pi 3 Model B+. A Raspberry Pi infrared camera is used to capture the picture at the front door and provide a real-time video streaming. First motion detection is performed on the streaming video using OpenCV. OpenCV compares two consecutive picture frames taken by the camera and uses the difference to determine whether there is a motion or not. If motion is detected, a picture will be taken instantaneously, then facial recognition is run to determine whether this person is authorized to enter or not. This motion-triggered facial recognition is implemented using a deep learning TensorFlow Lite model pretrained on a Windows computer. 


\subsection{Performance Evaluation}

\subsubsection{Accuracy for Facial Recognition}

To apply transfer learning for facial recognition, we compared several existing popular models for object detection. Single Shot Detection (SSD) is a fast object detection algorithm which takes one single shot to detect multiple objects in an image. MobileNet is designed and optimized for mobile phone and embedded device. It has been trained on COCO dataset (COCO 2020) for object detection. Faster-RCNN (Ren, 2017) model performs detection on multiple regions. It has a slower detection speed, but the accuracy is higher than the SSD-MobileNet model (Howard, 2017). Since SSD-MobileNet model is more suitable for devices with low computing power, such as mobile phones and Raspberry Pi, SSD-MobileNet V2 Quantized model trained on COCO dataset is chosen as the pre-trained model. This quantized model employs 8-bit integer values rather than 32-bit floating values inside the neural network, which enables them to operate more expeditiously on GPUs.

We generated our own images from web camera as the training dataset and retrained the model using transfer learning. The layers of the pre-trained model are frozen and new dense layers are added. We retrained the model at a Windows 10 computer using TensorFlow GUP 1.12.0. Initially the accuracy is only 90.18\% which is not accurate enough for facial recognition. The low accuracy is also resulted from the quality of the image, such as insufficient lightening, person not facing the camera. The accuracy of the retrained facial recognition model is improved up to $99 \%$ when testing on selfie pictures. The quality of the picture contributes to the improved accuracy. The background in the picture is bright enough without too many objects or colors. Person in the picture also faces the camera.

After the facial recognition model is trained, it is converted to a TensorFlow Lite format and deployed at Raspberry Pi. At Raspberry Pi, an image will be captured by camera when motion is detected. Facial recognition algorithm will perform inference on the image to determine whether the person in the picture is authorized user or not.

\subsubsection{Resource Consumption}

To examine the efficiency of TensorFlow Lite on low-power devices, performance of using TensorFlow and TensorFlow Lite for facial recognition running on Raspberry Pi are compared. As can be seen in Table 1, both CPU temperature and CPU usage when running TensorFlow Lite are much lower than running TensorFlow, which is suitable for low-power edge devices. TensorFlow Lite also has a higher average frame processing rate and shorter detection time. The collected data proves that TensorFlow Lite can reduce CPU load, power consumption while achieving a faster detection. The average detection time of $1.11 \mathrm{~s}$ for facial recognition can provide a real-time response.

Table 1. Performance Comparison of TensorFlow and TensorFlow Lite

\begin{tabular}{lll}
\hline Parameters & TensorFlow & TensorFlow Lite \\
\hline CPU Temperature $\left({ }^{\circ} \mathrm{F}\right)$ & 143.11 & 134.41 \\
CPU Temperature $\left({ }^{\circ} \mathrm{C}\right)$ & 61.73 & 56.89 \\
CPU Usage $(\%)$ & 58.7 & 22.2 \\
Average Frame Rate $(\mathrm{fps})$ & 0.7 & 0.82 \\
Average Detection Time $(\mathrm{s})$ & 1.35 & 1.11 \\
\hline
\end{tabular}

\subsubsection{Delay of Image Uploading}

Data collected by sensors or cameras can be either uploaded to the cloud server or a local server for backup and analytics. To compare the delay between data processing at IoT edge device and Internet cloud server, we measured the uploading time of 100 images in the two scenarios using TCP protocol in Figure 3. Cloud storage Dropbox is used here as an example of cloud server. The average uploading time to local server is 15.49 milliseconds (Figure 3a), which is significantly lower than the average uploading time to Dropbox which is 1827 milliseconds (Figure 3b). It can be expected that the end-to-end facial recognition at edge device can provide a much faster response than cloud processing. 


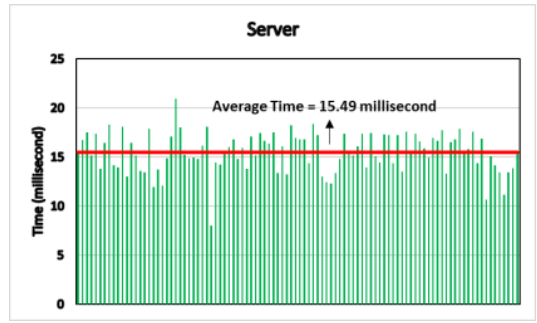

(a)

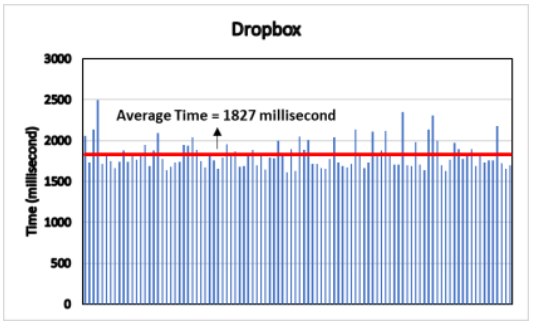

(b)

Figure 3. Comparison of Image Uploading Time

\section{CONCLUSION}

Applying machine learning to edge device can help improve the service provided in IoT applications. A prototype of IoT based home automation system is presented in this paper, which applies the deep learning intelligence at edge device. The performance evaluation demonstrates that edge computing diagram of IoT design can improve the quality of service of IoT system without relying on Internet connection. For future work, we will extend the technique to other IoT based monitoring applications. Besides applying the deep learning techniques to various object detection applications, we will also implement voice control and gesture recognition on microcontroller using TensorFlow Lite. The goal is to provide touchless interaction with physical devices in an IoT system.

\section{REFERENCES}

Arduino Nano 33 BLE, 2020, available at: https://store.arduino.cc/usa/nano-33-ble

COCO Dataset, 2020, available at https://cocodataset.org/\#home.

Google Vision API, available at: https://cloud.google.com/vision

Graves, A., et al, 2013, Speech recognition with deep recurrent neural networks. In 2013 IEEE international conference on acoustics, speech and signal processing, pp. 6645-6649.

Howard, A., et al, 2017. MobileNets: Efficient Convolutional Neural Networks for Mobile Vision Applications, arXiv:1704.04861.

Ren, S. et al, 2017. Fast R-CNN: Towards Real-time Object Detection with Region Proposal Networks, In IEEE Transactions on Pattern Analysis and Machine Intelligence, Vol. 39, No. 6, pp. 1137-1149.

Simonyan, K., et al, 2014, Very deep convolutional networks for large-scale image recognition, arXiv:1409.1556.

Szegedy, C., et al, 2015, Going Deeper with Convolutions, In 2015 IEEE Conference on Computer Vision and Pattern Recognition, pp. 1-9.

TensorFlow Lite, 2020, Deploy Machine Learning Models on Mobile and IoT Devices, available at: Tensorflow.org/lite. TensorFlow, 2020, available at: tensorflow.org

Wang X. et al, 2020. Convergence of Edge Computing and Deep Learning: A Comprehensive Survey, In IEEE Communications Survey \& Tutorials, Vol. 22, No. 2, pp 869-904. 